\title{
Un método para la identificación de tres especies crípticas de Protesilaus, (Lepidoptera: Papilionidae) del sur de Brasil, basado en su morfología genital
}

\author{
Luis Ricardo Murillo-Hiller \\ Museo de Insectos, Escuela de Agronomía, Universidad de Costa Rica, San José, Costa Rica; murillo.luis@lycos.com
}

Recibido 21-VI-2004. Corregido 26-I-2007. Aceptado 28-II-2007.

\begin{abstract}
A method to identify the three cryptic species of Protesilaus, (Lepidoptera: Papilionidae) from southern Brazil, based on genital morphology. I describe the male genital structures of three species of butterflies from Rio Grande do Sul, Brazil. Their external patterns do not differ enough to determine one species from another. All inhabit the same areas at the same time of the year. In an attempt to find a constant difference among them, as well as to establish the range of variability in each species, series of butterflies from different localities were dissected. The diagnostic structures found in all three species were named and drawn for their identification. Color patterns, width, separateness and presence or absence of the black bands and the color of the frons are not useful for recognizing $P$. nigricornis from $P$. stenodesmus or $P$. helios. The species group studied shows major morphological variability. For that reason, species level identification can be extremely difficult, particularly if the specimens are deteriorated. In conclusion, the only reliable way to correctly identify this species is by dissection of male genitalia. Rev. Biol. Trop. 55 (2): 665-671. Epub 2007 June, 29.
\end{abstract}

Key words: Eurytides, genitalia, sympatry, P. helios, P. stenodesmus, P. nigricornis.

El género Protesilaus (Swainson, 1832), comprende mariposas de tamaño mediano, alas de color blanco, un poco translúcido en las anteriores, con rayas negras atravezándolas longitudinalmente y una elongación de la vena M3 en las alas posteriores, unas dos veces más larga que sus antenas. Se encuentra ampliamente distribuido desde México hasta Argentina. Su vuelo es rápido y errático, por lo que su observación detallada en la naturaleza es difícil. Este género comprende diez especies, todas con el mismo patrón de coloración y tamaño similar. Presentan una gran variabilidad, principalmente en sus ornamentaciones negras. Por otra parte, algunas especies pueden presentar distribución simpátrica. Todos estos factores provocan que la identificación de las especies sea muchas veces difícil y subjetiva. Algunas especies del género, fueron estudiadas a nivel de órganos genitales por Zikán (1938, 1939) pero los resultados son confusos y con ilustraciones de poca calidad.

En el estado de Río Grande del Sur en Brasil, se han reportado cinco especies del género Protesilaus (Teston y Corseuil 1998), de las cuales solamente tres se han recolectado en la zona del noreste del estado: $P$. protesilaus nigricornis (Staudinger, 1884), P. stenodesmus (Rothschild y Jordan, 1906) y P. helios (Rothschild y Jordan, 1906), ésta última es la única que alcanza al centro y sur del estado (Krüger y Silva 2003). Estas a su vez, presentan épocas de vuelo similares en primavera, octubre, noviembre y diciembre (C. Agra, com. pers.).

A pesar de ser una familia muy conocida y estudiada, resulta inesperada la inexistencia de un método de identificación más preciso para 
estas especies. Por lo que el estudio de otras estructuras se hace imprescindible si se quiere llegar a conclusiones definitivas. Por tal motivo, se ha recurrido al estudio de los caracteres genitales de los machos, los cuales presentan una menor variabilidad, pues se presume que tienen funciones durante la cópula (Otero 1990), y además contribuyen en el papel de barrera de hibridización entre las especies con distribución simpátrica (Niculescu 1980).

El uso de estos caracteres, ha sido de gran ayuda en el pasado para resolver problemas de grupos de carácter parecido (Jenkins 1983, 1984, Steinhauser 1981, 1989).

El objetivo de este estudio es confirmar un método de identificación confiable para estas tres especies de Protesilaus del noreste del estado de Río Grande del Sur, por medio de la disección de los genitales de los machos.

\section{MATERIALES Y MÉTODOS}

Se hicieron recolectas de ejemplares en octubre, noviembre y diciembre del 2003, con el objetivo de obtener ejemplares frescos y bien conservados. Las capturas se realizaron en las localidades del morro Santana (MST), Porto Alegre y en la Floresta Nacional de San Francisco de Paula (FLONA) ubicado en el noreste del estado. Además, se estudiaron ejemplares de la colección de referencia de Lepidópteros del Laboratorio de Bioecología de Insectos del Departamento de Zoología en la Universidad Federal de Río Grande del Sur (LBIF) y del Museo de Ciencias Naturales de la Fundación Zoobotánica (MCNZ), Porto Alegre. En total fueron analizados diecisiete ejemplares: 12 de $P$. protesilaus nigricornis, tres de $P$. helios, y dos de $P$. stenodesmus. Las recolectas se realizaron con la ayuda de una red de golpe.

Para el estudio de los genitales, se removieron los últimos dos esternos del abdomen de los ejemplares y se sometieron a un tratamiento de digestión al calor con $\mathrm{KOH}$ a 10 $\%$. Seguidamente, se removió todo el material digerido con ayuda de un estereoscopio y una aguja de disección, hasta conservar solamente las estructuras esclerotizadas de los genitales, los cuales fueron guardados para su preservación en frascos de vidrio con alcohol al $70 \%$. Las ilustraciones fueron hechas con ayuda de fotografías de los genitales, tomadas con una cámara fotográfica acoplada al estereoscopio binocular. Los ejemplares testigo recolectados junto con sus genitales, se encuentran depositados en MCNZ y en LBIF.

\section{RESULTADOS}

A partir de los estudios de los genitales de los machos, se determinó que en las regiones MST y FLONA solo se recolectaron P. protesilaus nigricornis, la cual se puede considerar muy abundante. De las colecciones consultadas, se determinó que $P$. helios es una especie mucho menos abundante, teniéndose pocos registros de ella a lo largo de todo el este y centro del estado. Asimismo, P. stenodesmus también es una especie poco abundante, la cual se distribuye en el noreste del estado, donde las dos presentan una distribución simpátrica con P. protesilaus nigricornis.

Los genitales de las especies estudiadas (Fig. 1), se caracterizaron por presentar un saccus (s) corto e invariable, un Uncus (u) terminado en tres puntas también invariable. El aedeagus (ad) es muy similar en las tres especies. Por lo anterior, se determinó que únicamente los "brochers" ("claspers": cl) son informativos, los cuales presentan en la cara interna estructuras que varían de una especie a otra. Por tal razón, éstas fueron nombradas como proyección distal del "broche" (pdc), cresta dorsal del "broche" (cdc), proyección proximal del "broche" (ppc) y mano del "broche" (mc).

De las doce $P$. protesilaus nigricornis disectadas, se estableció un ámbito de variabilidad morfológica interna representativa para esta especie; que probablemente pueda ser tomada como modelo de la variación intraespecífica de las demás especies, que al ser poco abundante no existen muchos individuos de 


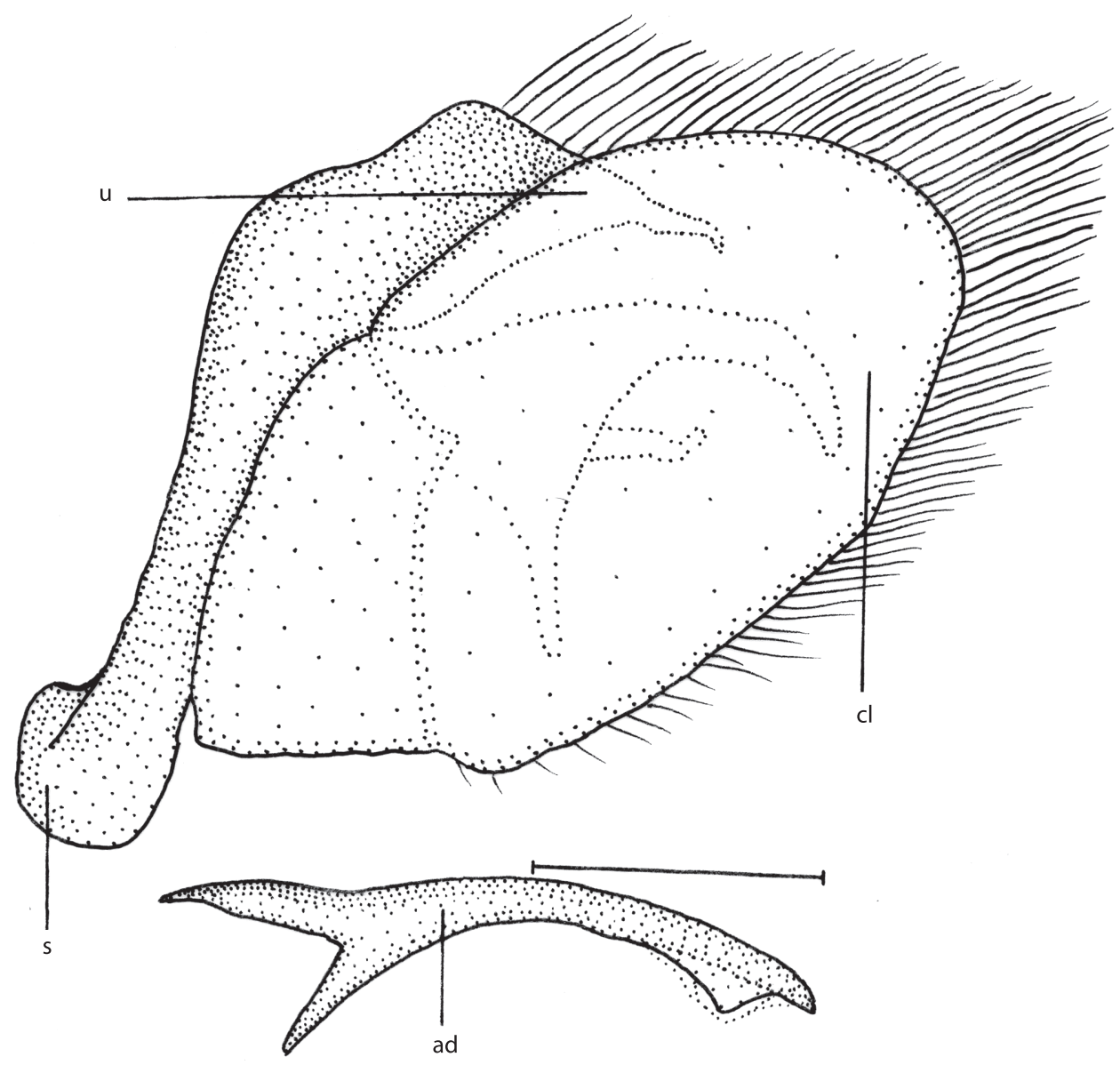

Fig. 1. Órganos genitales completos de macho, vista de perfíl. Uncus (u), saccus (s), "broche” (cl), aedeagus (ad). Escala: 1 $\mathrm{mm}$. (El resto del abdomen se encuentra hacia la izquierda).

Fig. 1. Complete genitalia of male, lateral view showing: uncus (u), saccus (s), clasper (cl), aedeagus (ad). Bar: $1 \mathrm{~mm}$. (Rest of the abdomen and body goes to the left side).

estas dos especies en colecciones. A partir de lo anterior se obtuvo que: el cl es muy variable en pubescencia y en la pdc aparecen espinas de diferentes tamaños y cantidades. La ppc está terminada en una o en dos puntas, sin embargo, puede presentar espinas variables. En síntesis, la diferencia entre las tres especies se encuentra en que $P$. protesilaus nigricornis presenta la cdc muy desarrollada, fuertemente esclerotizada y proyectada como una aleta de tiburón similar en tamaño como la pdc y completamente cubierta de grandes espinas (Fig. 2). En el caso de $P$. helios, ésta estructura se encuentra modificada en forma de sierra pequeña con espinas esclerotizadas de tamaño y altura similar (Fig. $3)$. Finalmente, en P. stenodesmus la estructura se encuentra totalmente ausente, dejando en su lugar una superficie lisa (Fig. 4). Por otro lado, se determinó que en la mc, $P$. protesilaus nigricornis presenta la terminación en espinas con forma de dedos, mientras que las otras dos especies termina en una punta lisa.

Material examinado de $P$. helios y $P$. stenodesmus:

\section{P. helios}

Rio Grande do Sul: Canguçu, Serra do Sudeste, 200 m.s.n.m. 16/X/2003, Gomes A.L. col. (LBIF). 


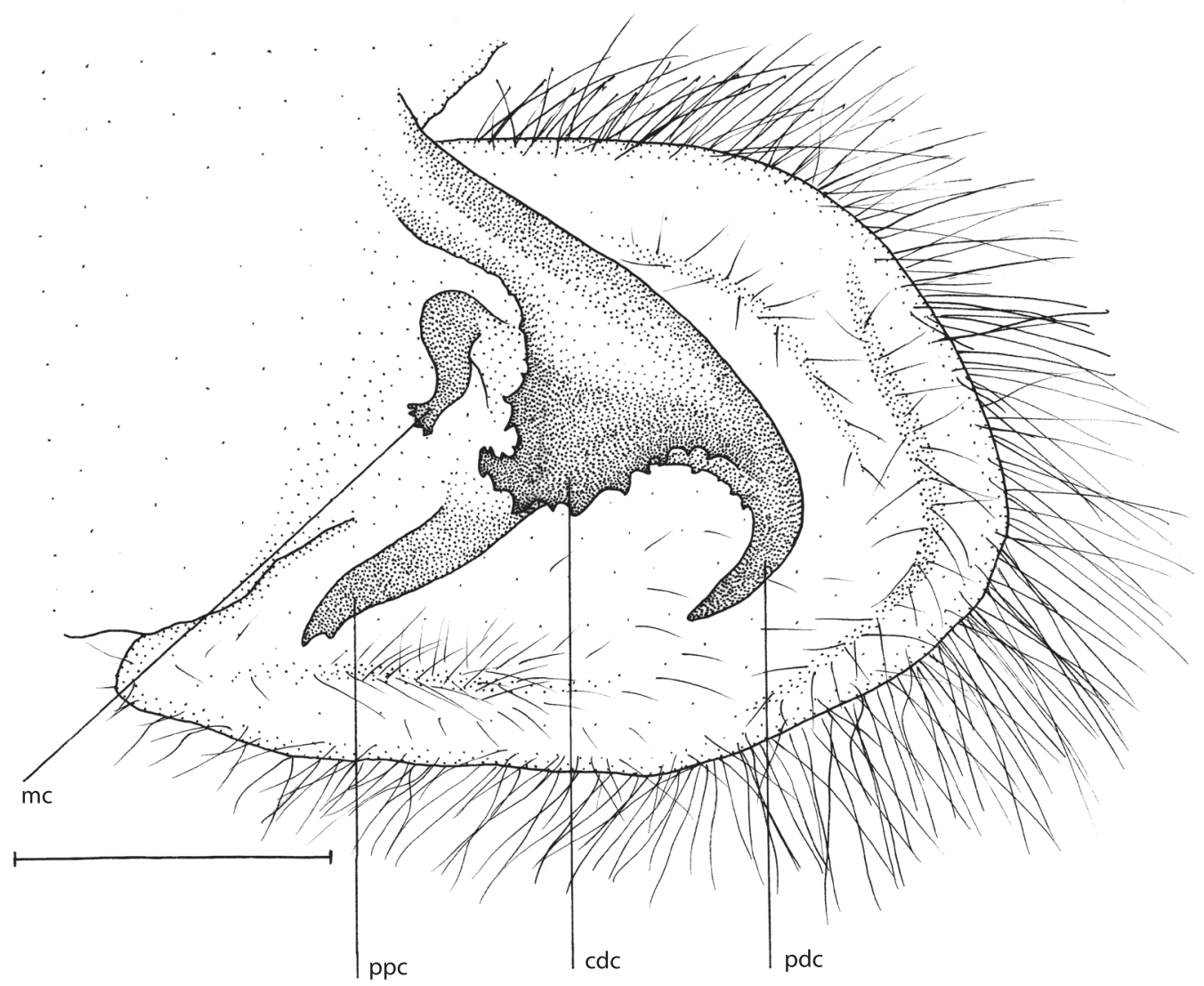

Fig. 2. Vista interna del "broche" derecho de P. protesilaus nigricornis. Mano (mc), proyección proximal (ppc), cresta dorsal (cdc), proyección distal (pdc). Escala: $1 \mathrm{~mm}$. (El resto del abdomen se encuentra hacia la izquierda).

Fig. 2. Right clasper internal view of $P$. protesilaus nigricornis. Hand of the clasper (mc), proximal projection of the clasper (ppc), dorsal crest of the clasper (cdc), distal projection of the clasper (pdc). Bar: $1 \mathrm{~mm}$. (Rest of the abdomen and body goes to the left side).

Rio Grande do Sul: Itaimbézinho, Cambará do Sul, 25/I/1959, Ditadi S. col. (LBIF)

Rio Grande do Sul: Caxias, 24/I/1927, (MCNZ).

\section{P. stenodesmus}

Rio Grande do Sul: Maquiné, 19/X/2001, Agra C. col. (LBIF).

Rio Grande do Sul: Oswaldo Cruz, Palnusina, 25/IV/1946, (MCNZ).

\section{DISCUSIÓN}

Debido al número de especies y a la gran variabilidad que éstas presentan, la identificación de estas mariposas se ha convertido en una tarea muy confusa. En años anteriores, varias especies han sido descritas por error en la interpretación de la variabilidad de los caracteres externos, e incluso de los internos, como es el caso de las especies descritas 


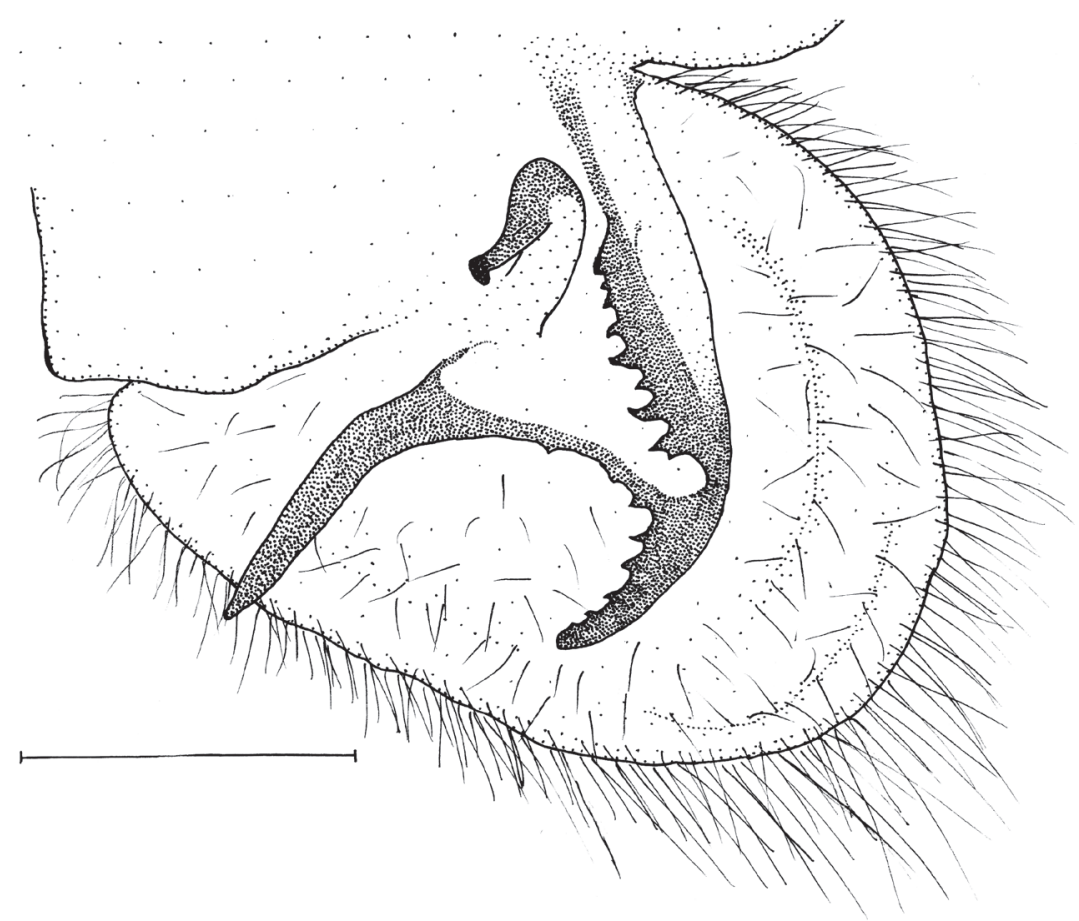

Fig. 3. Vista interna del "broche" derecho de P. helios. Escala: $1 \mathrm{~mm}$. (El resto del abdomen se encuentra hacia la izquierda). Fig. 3. Right clasper internal view of $P$. helios. Bar: $1 \mathrm{~mm}$. (Rest of the abdomen and body goes to the left side).

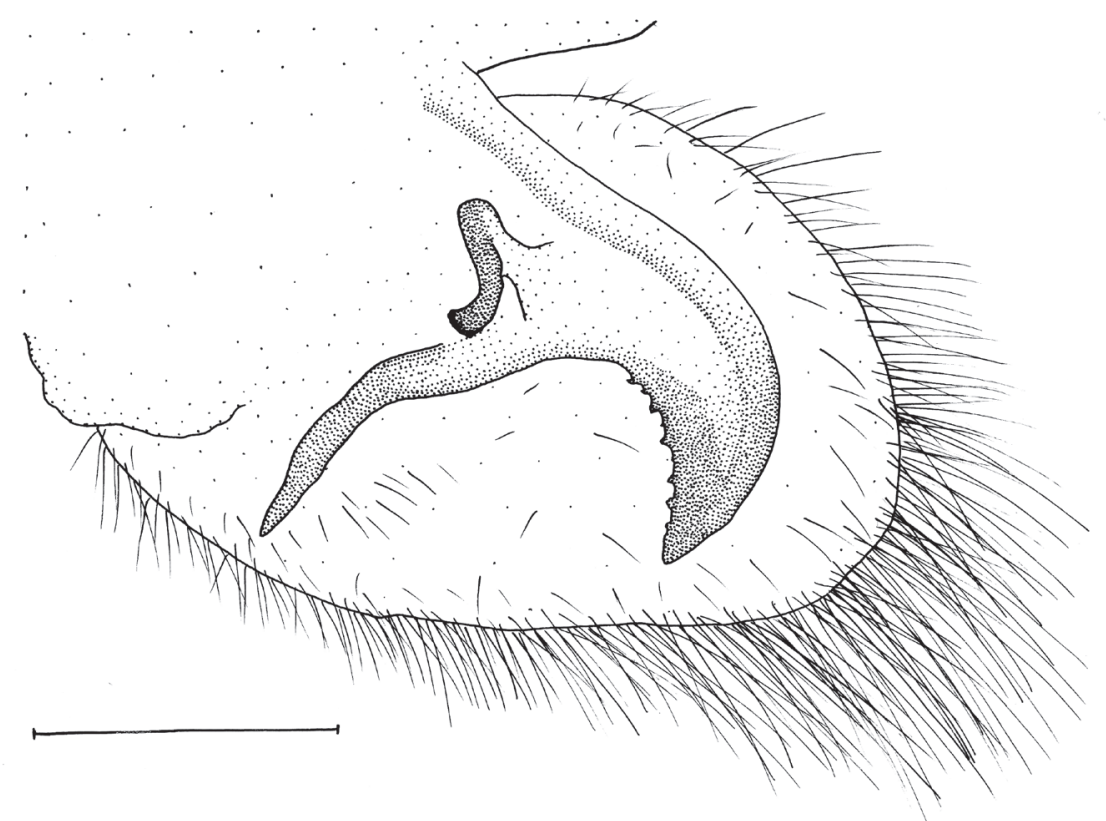

Fig. 4. Vista interna del "broche" derecho de P. stenodesmus. Escala: $1 \mathrm{~mm}$. (El resto del abdomen se encuentra hacia la izquierda).

Fig. 4. Right clasper internal view of P. stenodesmus. Bar: $1 \mathrm{~mm}$. (Rest of the abdomen and body goes to the left side). 
por D’Almeida $(1936,1938)$ que aun con el conocimiento de los genitales no consideró la variación de la misma. En el presente estudio, se examinaron series de genitales y se determinaron los caracteres que pueden o no presentar variación. Basándose en la morfología externa, se desarrollaron claves de difícil interpretación como la de Tyler et al. (1994) que hablan de alas más o menos transparentes y franjas más o menos distanciadas. Del mismo modo se menciona en la clave que el frons (zona más anterior de la cabeza entre los ojos) es blanco en $P$. stenodesmus, negro $P$. helios y en $P$. protesilaus nigricornis no se menciona de qué color es. Sin embargo en este estudio, con el respaldo de series de individuos según los genitales, se observó que $P$. protesilaus nigricornis puede presentar las condiciones de color blanco como negro, o una mezcla de los dos colores en individuos de la misma población en el mismo momento. En P. stenodesmus, es posible encontrar individuos con el frons negro y por lo que puede ser confundida con $P$. protesilaus nigricornis. Con respecto a las franjas negras de las alas anteriores, D'Abrera (1981) menciona que en $P$. helios una de estas es siempre reducida a un solo punto, sin embargo también se observó, que esta varía en tamaño de un individuo a otro.

En el estudio de la serie de P. protesilaus nigricornis, se observa que los caracteres externos como: las franjas negras de las alas anteriores, el grosor de la franja negra sub-marginal de las alas posteriores y la mancha roja anal de las alas posteriores; no son criterio de separación de especies. Las escamas amarillentas alrededor de la zona sub-marginal a la altura de la vena M3 y el grosor de la franja negra sub-marginal ambas en las alas posteriores, son utilizadas como punto de separación de $P$. helios (Canals 2003). Sin embargo, en el presente estudio se comprobó lo contrario, al observarse también en algunos casos en $P$. stenodesmus.

Finalmente se postula que la búsqueda de una forma externa y rápida de identificar estas especies, es difícil según los aspectos estudiados. Se debe aceptar que el método convencional de identificar mariposas a primera vista, no va a ser posible en este caso y para obtener una identificación segura y confiable de un individuo, se debe recurrir a la disección de los genitales.

\section{AGRADECIMIENTOS}

Agradezco a Aldo Mellender de Araújo y a Emily Fung por la revisión del manuscrito. También a Helena Romanowski por permitirme trabajar en el LBIF, a Lucas A. Kaminski y C. Agra por su apoyo, críticas y obtención de bibliografía. Finalmente a todo el personal del LBIF que aportó siempre su valiosa ayuda.

\section{RESUMEN}

Analizo la estructura genital de los machos de tres especies de Protesilaus de Río Grande du Sul en Brasil, debido a que los caracteres morfológicos externos no proporcionan la información suficiente para identificarlas hasta el nivel de especie. Las tres especies se pueden encontrar en zonas de simpatría y en los mismos meses del año. Se nombraron y dibujaron las estructuras diagnósticas para cada una de las especies. Características morfológicas externas como el grosor, separación y presencia o ausencia de las bandas negras, y los colores en la cabeza, no son útiles para separar a $P$. nigricornis de $P$. stenodesmus y de $P$. helios. El grupo presenta una alta variabilidad morfológica, por lo que identificarlas a nivel de especie puede resultar difícil, principalmente si los especímenes se encuentran deteriorados. La identificación confiable se debe realizar mediante el estudio de los órganos genitales de los machos.

Palabras clave: Eurytides, órganos genitales, simpatría, $P$. helios, P. stenodesmus, p. nigricornis.

\section{REFERENCIAS}

Canals, G.R. 2003. Mariposas de Misiones. Colin Sharp, Buenos Aires, Argentina.

D’Abrera, B. 1981. Butterflies of the Neotropical Region. Part 1 Papilionidae \& Pieridae. Lansdowne, Melbourne, England.

D'Almeida, R.F. 1936. Une nouvelle espèce d'Iphiclides (Papilio) du Brésil. Festschrift für Prof. Dr. Embrikstrand, Rija, Brasil 1: 510-513. 
D’Almeida, R.F. 1938. Uma nova espécie do gênero Iphiclides. Livro Juvilar Prof. Travassos, Rio de Janeiro, Brasil.

Jenkins, D.W. 1983. Neotropical Nymphalidae I. Revision of Hamadryas. Bull. Allyn Mus. 81: 1-146.

Jenkins, D.W. 1984. Neotropical Nymphalidae II. Revision of Myscelia. Bull. Allyn Mus. 87: 1-64.

Krüger, C.P. \& E.J.E. Silva. 2003. Papilionoidea (Lepidoptera) de Pelotas e seus arrededores, Río Grande do Sul, Brasil. Entomol. Vect. 10: 31-45.

Niculescu, E.V. 1980. La Speciation dans genre Eurytides (Lepidóptera, Papilionidae). Dusenia 12: 25-28.

Otero, L.D. 1990. Estudio de algunos caracteres para su uso en la clasificación de Eurytelinae (Lepidoptera: Nymphalidae). Bol. Entomol. Venez. N.S. 5: $123-$ 138.
Steinhauser, S.R. 1981. A revision of the Proteus group of the genus Urbanus Hubner Lepidóptera: Hesperiidae. Bull. Allyn Mus. 62: 1-41.

Steinhauser, S.R. 1989. Taxonomic notes and descriptions of new taxa in the neotropical Hesperiidae. Part I. Pyrginae. Bull. Allyn Mus. 127: 1-70.

Teston, J.A. \& E. Corseuil. 1998. Lista documentada dos papilionídeos (Lepidóptera, Papilionidae) do Rio Grande do Sul, Brasil. Biociências 6: 81-94.

Tyler, H.A., K.S. Brown Jr. \& K.H. Wilson. 1994. Swallowtail Butterflies of the Americas: a study in Biological Dinamics, Ecological Diversity, Biosystematics and conservation. Scientific, Gainesville, Florida, EEUU.

Zikán, J.F. 1938-1939. Die Arten der Papilio protesilausGruppe. Ent. Rundsch. 55: 229-235, 281-286, 301305, 389-303, 441-444, 561-564, 607-609. 
